\title{
Design, Synthesis and Biological Evaluation of Novel Osthole Derivatives as Potential Agents for the Treatment of Cancer
}

\author{
Jing Liu" ${ }^{1}$, Qi He ${ }^{2}$, Jiaoli Ding ${ }^{2}$, Zhipeng Zhang ${ }^{2}$, Weixin Zhou ${ }^{2}$, Saisai Xie ${ }^{2, *}$ \\ ${ }^{1}$ School of Pharmacy, Jiangxi University of Traditional Chinese Medicine, Nanchang, PR China \\ ${ }^{2}$ National Pharmaceutical Engineering Center for Solid Preparation in Chinese Herbal Medicine, Jiangxi University of Traditional Chinese \\ Medicine, Nanchang, PR China \\ Email address: \\ sadytse@126.com (Saisai Xie) \\ ${ }^{*}$ Corresponding author
}

To cite this article:

Jing Liu, Qi He, Jiaoli Ding, Zhipeng Zhang, Weixin Zhou, Saisai Xie. Design, Synthesis and Biological Evaluation of Novel Osthole Derivatives as Potential Agents for the Treatment of Cancer. Biochemistry and Molecular Biology. Vol. 3, No. 4, 2018, pp. 56-62. doi: $10.11648 /$ j.bmb.20180304.11

Received: November 16, 2018; Accepted: December 6, 2018; Published: December 18, 2018

\begin{abstract}
Aim: Osthole, an ingredient of Traditional Chinese Medicine (TCM) from natural product Cnidium monnieri (L.) Cusson, showed many biological activities related to the treatment of cancer. However, the comparatively weak activity hinders its further application in clinical. Therefore, the aim of the present study was design and synthesis of a series of novel osthole derivatives by introducing different secondary amine groups at 7-O position of coumarin ring to improve its anticancer activity. Method: After the demethylation of osthole, the target derivatives 4a-1 were successfully synthesized through two steps in high yield. The structures of the synthesized compounds were confirmed by ${ }^{1} \mathrm{H}$ and ${ }^{13} \mathrm{C}$ NMR. The antiproliferative activity of these compounds was evaluated against four selected cancer cell lines (SGC7901, MCF-7, HCT116 and HepG2) using MTT method, and the most potent compound was selected for Hoechst 333258 staining assay to further investigate its possible mechanism on cancer cells. Results: Twelve osthole derivatives were synthesized and their structures were identified. Biological studies showed that most of them showed moderate to good growth inhibition against all the tested cancer cells. Especially, compound 41 displayed the most potent activity with $\mathrm{IC}_{50}$ values of $37.57 \mu \mathrm{M}, 25.12 \mu \mathrm{M}, 46.54 \mu \mathrm{M}$ and $18.39 \mu \mathrm{M}$ against SCG7901, HCT116, MCF-7 and HepG2, respectively, which was more potent than those of its parent compound osthole and the anticancer agent 5-Fu. The Hoechst 33258 staining assay revealed that compound 41 could induce cell apoptosis in HepG2 cells. Conclusion: The modification of osthole to improve its activity is feasible, and compound 41 can be considered as a potential agent for the treatment of cancer.
\end{abstract}

Keywords: Osthole, Anticancer Activity, Synthesis, Apoptosis, Coumarin

\section{Introduction}

Cancer is one of the major diseases that threatens human health characterized by uncontrolled cell proliferation and spread of malignant cells $[1,2]$. According to the World health organization (WHO) report, there is nearly 7.6 million people died of cancer per year, and the figure is expecting to reach 13 million by 2030 [3]. Although much progress has been made from the identification to the treatment of cancer, the factors like poor patient compliance, drug resistance and drug induced toxicities make successful treatment of cancer remain a challenge [4, 5]. Therefore, the search for new effective anticancer drugs is highly needed.

Natural products with their inherent ability to interact with biological targets have played an important role in drug discovery [6-8]. Especially in cancer therapy, it is roughly estimated that more than half of modern marketed drugs are natural products or their derivatives [9-10]. Osthole 1, a prenylated coumarin derivative, is extracted from Cnidium monnieri (L) Cusson, which has been clinically ingested as an important component of medicinal plants and herbs in Tradition Chinese Medicine (TCM) [11]. A lot of studies 
showed that osthole could inhibit cancer cell proliferation, prevent cell migration and invasion, and induce differentiation, apoptosis and cell cycle arrest [12]. Additionally, it could also prolong the survival days of tumor-bearing mice and suppressed tumor growth in vivo [13]. However, although osthole have so many promising profiles related to cancer treatment, the comparatively weak activity hampered its application in clinical development [14, 15]. Therefore, structural modification of osthole to improve its anticancer activity is highly needed. In this work a series of novel osthole derivatives by introducing diversified secondary amines to 7-O position was designed and synthesized. All synthesized compounds were evaluated for their antiproliferative activity on HepG2 (liver carcinoma), SGC7901 (gastric carcinoma), HCT-116 (colon cancer) and MCF-7 (breast carcinoma) cells. In addition, compound with the most potent activity was also selected for mechanism assay.

\section{Materials and Methods}

\subsection{Materials}

All chemical reagents used in synthesis were obtained from Sinopharm Chemical Reagent Co., Ltd. (China). The reactions were monitored by TLC on glass-packed precoated silica gel $\mathrm{GF}_{254}$ (Qingdao Haiyang Chemical Plant, Qingdao, China) plates. Column chromatography was performed on silica gel (90-150 $\mu \mathrm{m}$; Qingdao Marine Chemical Inc.). ${ }^{1} \mathrm{H}$ NMR spectra $(600 \mathrm{MHz})$ and ${ }^{13} \mathrm{C}$ NMR spectra $(151 \mathrm{MHz})$ were recorded on a Bruker ACF-600 spectrometer at $25^{\circ} \mathrm{C}$. Chemical shifts are reported in $\operatorname{ppm}(\delta)$ using the TMS as internal standard, and the coupling constants are reported in hertz $(\mathrm{Hz})$. The purity of all compounds for biological evaluation was confirmed to $>95 \%$ by analytical HPLC conducted on an Agilent 1200 HPLC System. The desired derivatives were synthesized according to procedure depicted in Figure 1.

\subsection{General Procedure for the Synthesis of Compounds}

\section{Synthesis of compound 2}

To a solution of $1(1.00 \mathrm{~g}, 4.09 \mathrm{mmol})$ in anhydrous $\mathrm{CH}_{2} \mathrm{Cl}_{2}(10 \mathrm{~mL}), \mathrm{BBr}_{3}(2.00 \mathrm{~mL})$ was added dropwise at -40 ${ }^{\circ} \mathrm{C}$ under $\mathrm{N}_{2}$. After the addition of $\mathrm{BBr}_{3}$, the resulting solution was warmed to r.t. and stirred for an additional $12 \mathrm{~h}$. Then, it was diluted with $\mathrm{CH}_{2} \mathrm{Cl}_{2}(10 \mathrm{~mL})$, cooled in an ice-bath and slowly quenched with $10 \%$ aq $\mathrm{NaHCO}_{3}$. The organic phase was washed with brine, dried $\left(\mathrm{Na}_{2} \mathrm{SO}_{4}\right)$, filtered and concentrated in vacuo. The residue was purified by silica gel chromatography using EtOAc/petrol (1:4) as eluent to obtain target compound 2 as white solid.

Synthesis of compounds $3 a-c$.

A mixture of $2(2.0 \mathrm{mmol})$ with suitable $\alpha$, $\omega$-dibromoalkanes $(50 \mathrm{mmol})$ and anhydrous $\mathrm{K}_{2} \mathrm{CO}_{3}(1.4 \mathrm{~g}$, $10 \mathrm{mmol})$ in acetone $(15 \mathrm{~mL})$ was refluxed under stirring for $4 \mathrm{~h}$. After cooling, the reaction mixture was filtered, and the filtrate was evaporated under reduced pressure. The obtained residue was purified by silica gel chromatography with EtOAc/petrol (1:7) as eluent to give compounds 3a-c as white solid.

Synthesis of compounds $4 a-l$.

A mixture of 3a-c $(1.2 \mathrm{mmol})$ and the corresponding secondary amines $(1.3 \mathrm{mmol})$ in dry acetonitrile $(10 \mathrm{~mL})$ was refluxed in the presence of anhydrous $\mathrm{K}_{2} \mathrm{CO}_{3}(1.3 \mathrm{mmol})$ for $8 \mathrm{~h}$. After cooling to the room temperature, the mixture was filtered, and the filtrate was evaporated under reduced pressure. The residue was purified by silica gel chromatography using $\mathrm{CH}_{2} \mathrm{Cl}_{2} / \mathrm{MeOH}$ (100:1) as eluent to obtain target compounds $4 \mathrm{a}-1$.

\subsection{Biological Evaluation}

\section{Cytotoxicity study}

In all the experiments, different cell lines were plated into 96-well plates at a concentration of $5 \times 10^{3}$ cells per well. After overnight incubation, the culture medium was removed and replaced with fresh medium containing the test compounds in different concentrations. The cells were incubated for another $48 \mathrm{~h}$. Afterward, $20 \mu \mathrm{L}$ of MTT solution $(5 \mathrm{mg} / \mathrm{mL})$ was added to each well and incubated for $4 \mathrm{~h}$ at 37 ${ }^{\circ} \mathrm{C}$. Then, the supernatant was aspirated and $150 \mu \mathrm{L}$ of dimethyl sulfoxide (DMSO) was added to dissolve the dark blue crystals (formazan crystals). After shaking for $20 \mathrm{~min}$, the absorbance was measured at a wavelength of $570 \mathrm{~nm}$ using a microplate reader (SpectraMax plus 384, Molecular Devices). Each concentration was analyzed in triplicate and the experiment was repeated three times. The average $50 \%$ inhibitory concentration $\left(\mathrm{IC}_{50}\right)$ was determined from the dose-response curves according to the inhibition ratio for each concentration.

\section{Hoechst 333258 staining}

Cells grown on a sterile cover slip in six-well tissue culture plates were treated with compounds for a certain range of time. The culture medium containing compounds was removed, and the cells were fixed in 4\% paraformaldehyde for $10 \mathrm{~min}$. After being washed twice with PBS, the cells were stained with 0.5 $\mathrm{mL}$ of Hoechst 33258 (Beyotime) for $5 \mathrm{~min}$ and then again washed twice with PBS. The stained nuclei were observed under an IX71SIF-3 fluorescence microscope using $350 \mathrm{~nm}$ excitation and $460 \mathrm{~nm}$ emission.

\section{Results and Discussion}

\subsection{Chemistry}

The synthetic routes for the preparation of compounds $4 \mathrm{a}-1$ are depicted in Figure 1. Osthole was demethylated by using boron tribromide etherate under $-40{ }^{\circ} \mathrm{C}$ to room temperature to afford compound 2 in high yield. Then, reacting the compound 2 with appropriate $\alpha, \omega$-dibromoalkanes to give compound 3 . Finally, compound 3 was coupled with the corresponding secondary amines to accomplish the target compounds 4a-l. The structures of all compounds were confirmed by NMR spectroscopy, and the detail data are shown in Table 1. 
Table 1. The NMR data of compounds 2, 3a-c and 4a-l.

\begin{tabular}{|c|c|c|}
\hline Compd. & Yield & ${ }^{1} \mathrm{H}$ and ${ }^{13} \mathrm{C}$ NMR data \\
\hline 2 & $92 \%$ & $\begin{array}{l}{ }^{1} \mathrm{H} \text { NMR }(600 \mathrm{MHz}, \mathrm{DMSO}) \delta 7.91(\mathrm{~d}, J=9.4 \mathrm{~Hz}, 1 \mathrm{H}), 7.37(\mathrm{~d}, J=8.5 \mathrm{~Hz}, 1 \mathrm{H}), 6.87(\mathrm{~d}, J=8.5 \mathrm{~Hz}, 1 \mathrm{H}), 6.19(\mathrm{~d}, J=8.7 \mathrm{~Hz}, \\
1 \mathrm{H}), 5.18(\mathrm{~m}, 1 \mathrm{H}), 3.37(\mathrm{~d}, J=5.2 \mathrm{~Hz}, 2 \mathrm{H}), 1.78(\mathrm{~s}, 3 \mathrm{H}), 1.62(\mathrm{~s}, 3 \mathrm{H}) .\end{array}$ \\
\hline $3 a$ & $86 \%$ & $\begin{array}{l}{ }^{1} \mathrm{H} \text { NMR }(600 \mathrm{MHz}, \mathrm{DMSO}) \delta 7.99(\mathrm{~d}, J=9.5 \mathrm{~Hz}, 1 \mathrm{H}), 7.57(\mathrm{~d}, J=8.6 \mathrm{~Hz}, 1 \mathrm{H}), 7.07(\mathrm{~d}, J=8.7 \mathrm{~Hz}, 1 \mathrm{H}), 6.31(\mathrm{~d}, J=9.5 \mathrm{~Hz}, \\
1 \mathrm{H}), 5.28-5.15(\mathrm{~m}, 1 \mathrm{H}), 4.51-4.38(\mathrm{~m}, 2 \mathrm{H}), 3.90-3.80(\mathrm{~m}, 2 \mathrm{H}), 3.47(\mathrm{~d}, J=7.4 \mathrm{~Hz}, 2 \mathrm{H}), 1.81(\mathrm{~s}, 3 \mathrm{H}), 1.63(\mathrm{~s}, 3 \mathrm{H}) .\end{array}$ \\
\hline $3 b$ & $88 \%$ & $\begin{array}{l}{ }^{1} \mathrm{H} \text { NMR }(600 \mathrm{MHz}, \mathrm{DMSO}) \delta 8.01(\mathrm{~d}, J=9.5 \mathrm{~Hz}, 1 \mathrm{H}), 7.59(\mathrm{~d}, J=8.6 \mathrm{~Hz}, 1 \mathrm{H}), 7.08(\mathrm{~d}, J=8.7 \mathrm{~Hz}, 1 \mathrm{H}), 6.32(\mathrm{~d}, J=9.5 \mathrm{~Hz}, \\
1 \mathrm{H}), 5.26-5.16(\mathrm{~m}, 1 \mathrm{H}), 4.44(\mathrm{t}, J=6.0 \mathrm{~Hz}, 2 \mathrm{H}), 3.84(\mathrm{t}, J=6.4 \mathrm{~Hz}, 2 \mathrm{H}), 3.47(\mathrm{~d}, J=7.4 \mathrm{~Hz}, 2 \mathrm{H}), 2.42-2.36(\mathrm{~m}, 2 \mathrm{H}), 1.82(\mathrm{~s}, \\
3 \mathrm{H}), 1.62(\mathrm{~s}, 3 \mathrm{H}) .\end{array}$ \\
\hline $3 \mathrm{c}$ & $83 \%$ & $\begin{array}{l}{ }^{1} \mathrm{H} \text { NMR }(600 \mathrm{MHz}, \mathrm{DMSO}) \delta 7.98(\mathrm{~d}, J=9.5 \mathrm{~Hz}, 1 \mathrm{H}), 7.58(\mathrm{~d}, J=8.6 \mathrm{~Hz}, 1 \mathrm{H}), 7.09(\mathrm{~d}, J=8.7 \mathrm{~Hz}, 1 \mathrm{H}), 6.32(\mathrm{~d}, J=9.5 \mathrm{~Hz}, \\
1 \mathrm{H}), 5.28-5.17(\mathrm{~m}, 1 \mathrm{H}), 4.32(\mathrm{t}, J=6.0 \mathrm{~Hz}, 2 \mathrm{H}), 3.67(\mathrm{t}, J=6.0 \mathrm{~Hz}, 2 \mathrm{H}), 3.47(\mathrm{~d}, J=7.4 \mathrm{~Hz}, 2 \mathrm{H}), 2.32-2.16(\mathrm{~m}, 2 \mathrm{H}), 2.17- \\
2.06(\mathrm{~m}, 2 \mathrm{H}), 1.80(\mathrm{~s}, 3 \mathrm{H}), 1.62(\mathrm{~s}, 3 \mathrm{H}) .\end{array}$ \\
\hline $4 a$ & $76 \%$ & $\begin{array}{l}{ }^{1} \mathrm{H} \mathrm{NMR}\left(600 \mathrm{MHz}, \mathrm{CDCl}_{3}\right) \delta 7.61(\mathrm{~d}, J=9.4 \mathrm{~Hz}, 1 \mathrm{H}), 7.28(\mathrm{~s}, 1 \mathrm{H}), 6.83(\mathrm{~d}, J=8.6 \mathrm{~Hz}, 1 \mathrm{H}), 6.24(\mathrm{~d}, J=9.4 \mathrm{~Hz}, 1 \mathrm{H}), 5.23(\mathrm{~d}, J \\
=1.2 \mathrm{~Hz}, 1 \mathrm{H}), 4.18(\mathrm{t}, J=5.9 \mathrm{~Hz}, 2 \mathrm{H}), 3.55(\mathrm{~d}, J=7.2 \mathrm{~Hz}, 2 \mathrm{H}), 2.81(\mathrm{t}, J=5.9 \mathrm{~Hz}, 2 \mathrm{H}), 2.37(\mathrm{~s}, 6 \mathrm{H}), 1.84(\mathrm{~s}, 3 \mathrm{H}), 1.66(\mathrm{~s}, 3 \mathrm{H}) . \\
{ }^{13} \mathrm{C} \mathrm{NMR}\left(151 \mathrm{MHz}, \mathrm{CDCl}_{3}\right) \delta 161.36,159.34,152.94,143.72,132.51,126.22,121.24,118.18,113.14,108.23,67.30,58.12, \\
46.02,25.79,22.07,18.07 .\end{array}$ \\
\hline $4 b$ & $79 \%$ & $\begin{array}{l}{ }^{1} \mathrm{H} \text { NMR }\left(600 \mathrm{MHz}, \mathrm{CDCl}_{3}\right) \delta 7.63(\mathrm{~d}, J=9.1 \mathrm{~Hz}, 1 \mathrm{H}), 7.29(\mathrm{~d}, J=5.3 \mathrm{~Hz}, 1 \mathrm{H}), 6.85(\mathrm{~d}, J=8.3 \mathrm{~Hz}, 1 \mathrm{H}), 6.26(\mathrm{~d}, J=9.3 \mathrm{~Hz}, \\
1 \mathrm{H}), 5.26(\mathrm{~s}, 1 \mathrm{H}), 4.16(\mathrm{~s}, 2 \mathrm{H}), 3.57(\mathrm{~d}, J=6.6 \mathrm{~Hz}, 2 \mathrm{H}), 2.95(\mathrm{~s}, 2 \mathrm{H}), 2.75-2.59(\mathrm{~m}, 4 \mathrm{H}), 1.86(\mathrm{~s}, 3 \mathrm{H}), 1.69(\mathrm{~s}, 3 \mathrm{H}), 1.11(\mathrm{~s}, 6 \mathrm{H}) \text {. } \\
{ }^{13} \mathrm{C} \text { NMR }\left(151 \mathrm{MHz}, \mathrm{CDCl}_{3}\right) \delta 161.40,159.51,152.92,143.75,132.48,128.81,126.19,121.27,118.09,113.02,108.25,67.74, \\
51.82,47.93,25.79,22.04,18.07,11.95 .\end{array}$ \\
\hline $4 c$ & $82 \%$ & $\begin{array}{l}{ }^{1} \mathrm{H} \text { NMR }\left(600 \mathrm{MHz}, \mathrm{CDCl}_{3}\right) \delta 7.53(\mathrm{~d}, J=9.0 \mathrm{~Hz}, 1 \mathrm{H}), 7.19(\mathrm{~d}, J=8.9 \mathrm{~Hz}, 1 \mathrm{H}), 6.75(\mathrm{~d}, J=8.5 \mathrm{~Hz}, 1 \mathrm{H}), 6.15(\mathrm{~d}, J=9.4 \mathrm{~Hz} \\
1 \mathrm{H}), 5.16(\mathrm{~s}, 1 \mathrm{H}), 4.17-4.06(\mathrm{~m}, 2 \mathrm{H}), 3.47(\mathrm{~d}, J=7.1 \mathrm{~Hz}, 2 \mathrm{H}), 2.93-2.81(\mathrm{~m}, 2 \mathrm{H}), 2.58(\mathrm{~s}, 4 \mathrm{H}), 1.77-1.68(\mathrm{~m}, 6 \mathrm{H}), 1.65- \\
1.52(\mathrm{~m}, 4 \mathrm{H}) .{ }^{13} \mathrm{C} \mathrm{NMR}\left(151 \mathrm{MHz}, \mathrm{CDCl}_{3}\right) \delta 161.38,159.43,152.94,143.75,132.46,128.81,126.22,121.31,118.11,113.04 \\
108.23,68.16,54.91,54.80,25.79,23.59,22.06,18.08\end{array}$ \\
\hline $4 d$ & $78 \%$ & $\begin{array}{l}{ }^{1} \mathrm{H} \text { NMR }\left(600 \mathrm{MHz}, \mathrm{CDCl}_{3}\right) \delta 7.61(\mathrm{~d}, J=9.4 \mathrm{~Hz}, 1 \mathrm{H}), 7.28(\mathrm{~d}, J=8.1 \mathrm{~Hz}, 1 \mathrm{H}), 6.83(\mathrm{~d}, J=8.6 \mathrm{~Hz}, 1 \mathrm{H}), 6.24(\mathrm{~d}, J=9.4 \mathrm{~Hz}, \\
1 \mathrm{H}), 5.25-5.20(\mathrm{~m}, 1 \mathrm{H}), 4.22(\mathrm{t}, J=5.9 \mathrm{~Hz}, 2 \mathrm{H}), 3.55(\mathrm{~d}, J=7.2 \mathrm{~Hz}, 2 \mathrm{H}), 2.85(\mathrm{t}, J=5.9 \mathrm{~Hz}, 2 \mathrm{H}), 2.56(\mathrm{br} \mathrm{s}, 4 \mathrm{H}), 1.85(\mathrm{~s}, 3 \mathrm{H}), \\
\left.1.67(\mathrm{~s}, 3 \mathrm{H}), 1.66-1.60(\mathrm{~m}, 4 \mathrm{H}), 1.47(\mathrm{br} \mathrm{s}, 2 \mathrm{H}) .{ }^{13} \mathrm{C} \mathrm{NMR}(151 \mathrm{MHz}, \mathrm{CDCl})_{3}\right) \delta 161.38,159.35,152.92,143.74,132.51,126.23 \text {, } \\
121.27,118.10,113.09,110.10,108.25,66.99,57.76,55.11,25.81,23.98,22.07,18.10 .\end{array}$ \\
\hline $4 \mathrm{e}$ & $84 \%$ & $\begin{array}{l}{ }^{1} \mathrm{H} \text { NMR }\left(600 \mathrm{MHz}, \mathrm{CDCl}_{3}\right) \delta 7.61(\mathrm{~d}, J=9.5 \mathrm{~Hz}, 1 \mathrm{H}), 7.28(\mathrm{~d}, J=8.6 \mathrm{~Hz}, 1 \mathrm{H}), 6.82(\mathrm{~d}, J=8.6 \mathrm{~Hz}, 1 \mathrm{H}), 6.25(\mathrm{~d}, J=9.4 \mathrm{~Hz}, \\
1 \mathrm{H}), 5.25-5.16(\mathrm{~m}, 1 \mathrm{H}), 4.22(\mathrm{~s}, 2 \mathrm{H}), 3.81-3.66(\mathrm{~m}, 4 \mathrm{H}), 3.54(\mathrm{~d}, J=7.2 \mathrm{~Hz}, 2 \mathrm{H}), 2.87(\mathrm{~s}, 2 \mathrm{H}), 2.62(\mathrm{~s}, 4 \mathrm{H}), 1.83(\mathrm{~s}, 3 \mathrm{H}), 1.67 \\
(\mathrm{~s}, 3 \mathrm{H}) .{ }^{13} \mathrm{C} \mathrm{NMR}\left(151 \mathrm{MHz}, \mathrm{CDCl}_{3}\right) \delta 161.10,159.22,152.94,143.68,132.68,126.24,121.24,118.17,113.23,108.18,66.84 \\
57.54,54.08,25.30,22.08,18.11 .\end{array}$ \\
\hline $4 f$ & $86 \%$ & $\begin{array}{l}{ }^{1} \mathrm{H} \text { NMR }\left(600 \mathrm{MHz}, \mathrm{CDCl}_{3}\right) \delta 7.54(\mathrm{~d}, J=7.8 \mathrm{~Hz}, 1 \mathrm{H}), 7.31-7.17(\mathrm{~m}, 1 \mathrm{H}), 6.74(\mathrm{~d}, J=8.6 \mathrm{~Hz}, 1 \mathrm{H}), 6.16(\mathrm{~d}, J=9.4 \mathrm{~Hz}, 1 \mathrm{H}), 5.15 \\
(\mathrm{~d}, J=7.0 \mathrm{~Hz}, 1 \mathrm{H}), 4.12(\mathrm{t}, J=5.7 \mathrm{~Hz}, 2 \mathrm{H}), 3.46(\mathrm{~d}, J=7.1 \mathrm{~Hz}, 2 \mathrm{H}), 2.80(\mathrm{t}, J=5.7 \mathrm{~Hz}, 2 \mathrm{H}), 2.40(\mathrm{br} \mathrm{s}, 8 \mathrm{H}), 2.22(\mathrm{~s}, 3 \mathrm{H}), 1.78(\mathrm{~s}, \\
3 \mathrm{H}), 1.59(\mathrm{~s}, 3 \mathrm{H}) .{ }^{13} \mathrm{C} \mathrm{NMR}\left(151 \mathrm{MHz}, \mathrm{CDCl}_{3}\right) \delta 161.34,159.38,152.90,143.74,132.46,126.21,121.25,118.12,113.05,108.21 \text {, } \\
67.11,57.04,55.09,53.60,45.99,25.80,22.05,18.10 .\end{array}$ \\
\hline $4 \mathrm{~g}$ & $76 \%$ & $\begin{array}{l}{ }^{1} \mathrm{H} \text { NMR }\left(600 \mathrm{MHz}, \mathrm{CDCl}_{3}\right) \delta 7.64(\mathrm{~d}, J=9.0 \mathrm{~Hz}, 1 \mathrm{H}), 7.47(\mathrm{~d}, J=8.4 \mathrm{~Hz}, 1 \mathrm{H}), 6.76(\mathrm{~d}, J=9.0 \mathrm{~Hz}, 1 \mathrm{H}), 6.17(\mathrm{~d}, J=9.4 \mathrm{~Hz}, 1 \mathrm{H}), \\
5.19-5.12(\mathrm{~m}, 1 \mathrm{H}), 4.05(\mathrm{t}, J=6.3 \mathrm{~Hz}, 2 \mathrm{H}), 3.47(\mathrm{~d}, J=7.2 \mathrm{~Hz}, 2 \mathrm{H}), 2.67-2.29(\mathrm{~m}, 10 \mathrm{H}), 2.25(\mathrm{~s}, 3 \mathrm{H}), 1.99-1.96(\mathrm{~m}, 2 \mathrm{H}), 1.77 \\
(\mathrm{~s}, 3 \mathrm{H}), 1.61(\mathrm{~s}, 3 \mathrm{H}) .{ }^{13} \mathrm{C} \text { NMR }\left(151 \mathrm{MHz}, \mathrm{CDCl}_{3}\right) \delta 161.41,159.57,152.93,143.76,132.44,128.83,126.19,121.33,118.02, \\
113.00,108.15,68.18,54.91,54.78,52.66,45.63,28.95,25.84,22.04,18.10 .\end{array}$ \\
\hline $4 \mathrm{~h}$ & $74 \%$ & $\begin{array}{l}{ }^{1} \mathrm{H} \text { NMR }\left(600 \mathrm{MHz}, \mathrm{CDCl}_{3}\right) \delta 7.63(\mathrm{~d}, J=7.2 \mathrm{~Hz}, 1 \mathrm{H}), 7.50(\mathrm{~d}, J=7.5 \mathrm{~Hz}, 1 \mathrm{H}), 6.82(\mathrm{~d}, J=9.0 \mathrm{~Hz}, 1 \mathrm{H}), 6.20(\mathrm{~d}, J=9.6 \mathrm{~Hz} \\
1 \mathrm{H}), 5.16(\mathrm{t}, J=7.2 \mathrm{~Hz}, 1 \mathrm{H}), 4.16-4.13(\mathrm{~m}, 2 \mathrm{H}), 4.01(\mathrm{~m}, 2 \mathrm{H}), 3.47(\mathrm{~d}, J=7.2 \mathrm{~Hz}, 2 \mathrm{H}), 2.40(\mathrm{br} \mathrm{s}, 8 \mathrm{H}), 2.22(\mathrm{~s}, 3 \mathrm{H}), 1.81- \\
1.75(\mathrm{~m}, 5 \mathrm{H}), 1.65-1.59(\mathrm{~m}, 5 \mathrm{H}) .{ }^{13} \mathrm{C} \mathrm{NMR}\left(151 \mathrm{MHz}, \mathrm{CDCl}_{3}\right) \delta 161.42,159.61,152.92,143.76,132.46,128.81,126.17 \\
121.29,117.97,112.94,108.09,68.16,58.02,54.91,52.85,38.73,30.37,28.93,27.26,25.81,23.75,22.04,18.03\end{array}$ \\
\hline $4 \mathrm{i}$ & $68 \%$ & $\begin{array}{l}{ }^{1} \mathrm{H} \text { NMR }\left(600 \mathrm{MHz}, \mathrm{CDCl}_{3}\right) \delta 7.62(\mathrm{~d}, J=9.4 \mathrm{~Hz}, 1 \mathrm{H}), 7.55(\mathrm{~d}, J=8.4 \mathrm{~Hz}, 1 \mathrm{H}), 6.83(\mathrm{~d}, J=8.6 \mathrm{~Hz}, 1 \mathrm{H}), 6.25(\mathrm{~d}, J=9.4 \mathrm{~Hz} \\
1 \mathrm{H}), 5.24(\mathrm{~s}, 1 \mathrm{H}), 4.28-4.17(\mathrm{~m}, 2 \mathrm{H}), 3.56(\mathrm{~d}, J=7.2 \mathrm{~Hz}, 2 \mathrm{H}), 2.90(\mathrm{t}, J=5.7 \mathrm{~Hz}, 2 \mathrm{H}), 2.66(\mathrm{br} \mathrm{s}, 9 \mathrm{H}), 1.85(\mathrm{~s}, 3 \mathrm{H}), 1.71(\mathrm{~s}, \\
3 \mathrm{H}), 1.07(\mathrm{br} \mathrm{s}, 6 \mathrm{H}) .{ }^{13} \mathrm{C} \mathrm{NMR}\left(151 \mathrm{MHz}, \mathrm{CDCl}^{3}\right) \delta 161.35,159.38,152.93,143.72,132.46,128.81,126.20,121.25,118.16 \\
113.10,108.19,68.16,67.06,65.20,57.03,54.74,53.69,25.81,23.75,22.99,22.07,18.12\end{array}$ \\
\hline $4 j$ & $84 \%$ & $\begin{array}{l}{ }^{1} \mathrm{H} \text { NMR }\left(600 \mathrm{MHz}, \mathrm{CDCl}_{3}\right) \delta 7.62(\mathrm{~d}, J=9.6 \mathrm{~Hz}, 1 \mathrm{H}), 7.28(\mathrm{~d}, J=8.4 \mathrm{~Hz}, 1 \mathrm{H}), 6.82(\mathrm{~d}, J=8.6 \mathrm{~Hz}, 1 \mathrm{H}), 6.25(\mathrm{~d}, J=9.4 \mathrm{~Hz}, \\
1 \mathrm{H}), 5.30-5.16(\mathrm{~m}, 1 \mathrm{H}), 4.22(\mathrm{t}, J=5.7 \mathrm{~Hz}, 2 \mathrm{H}), 3.54(\mathrm{~d}, J=7.2 \mathrm{~Hz}, 2 \mathrm{H}), 2.89(\mathrm{t}, J=5.7 \mathrm{~Hz}, 2 \mathrm{H}), 2.67(\mathrm{br} \mathrm{s}, 9 \mathrm{H}), 1.84(\mathrm{~s}, 3 \mathrm{H}), \\
1.67(\mathrm{~s}, 3 \mathrm{H}), 0.47-0.43(\mathrm{~m}, 4 \mathrm{H}) .{ }^{13} \mathrm{C} \mathrm{NMR}\left(151 \mathrm{MHz}, \mathrm{CDCl}_{3}\right) \delta 161.36,159.34,152.93,143.72,132.52,126.20,121.25,118.16 \text {, } \\
113.12,108.19,66.25,57.08,53.37,53.06,38.44,25.32,21.85,18.02,5.51 .\end{array}$ \\
\hline $4 k$ & $85 \%$ & $\begin{array}{l}{ }^{1} \mathrm{H} \text { NMR }\left(600 \mathrm{MHz}, \mathrm{CDCl}_{3}\right) \delta 8.25-8.24(\mathrm{~m}, 2 \mathrm{H}), 7.55(\mathrm{~d}, J=9.4 \mathrm{~Hz}, 1 \mathrm{H}), 7.24-7.20(\mathrm{~m}, 1 \mathrm{H}), 6.77(\mathrm{~d}, J=8.6 \mathrm{~Hz}, 1 \mathrm{H}), 6.43 \\
(\mathrm{t}, J=4.7 \mathrm{~Hz}, 1 \mathrm{H}), 6.18(\mathrm{~d}, J=9.4 \mathrm{~Hz}, 1 \mathrm{H}), 5.16(\mathrm{~s}, 1 \mathrm{H}), 4.20(\mathrm{br} \mathrm{s}, 2 \mathrm{H}), 3.80(\mathrm{br} \mathrm{s}, 4 \mathrm{H}), 3.48(\mathrm{~d}, J=7.2 \mathrm{~Hz}, 2 \mathrm{H}), 2.88(\mathrm{br} \mathrm{s}, 2 \mathrm{H}), \\
2.62(\mathrm{br} \mathrm{s}, 4 \mathrm{H}), 1.77(\mathrm{~s}, 3 \mathrm{H}), 1.60(\mathrm{~s}, 3 \mathrm{H}) .{ }^{13} \mathrm{C} \text { NMR }\left(151 \mathrm{MHz}, \mathrm{CDCl}_{3}\right) \delta 161.58,161.26,159.15,157.78,152.97,143.69,132.64 \text {, } \\
126.26,121.20,118.19,113.26,108.23,66.75,57.16,54.75,53.53,25.78,22.03,18.09 .\end{array}$ \\
\hline 41 & $83 \%$ & $\begin{array}{l}{ }^{1} \mathrm{H} \text { NMR }\left(600 \mathrm{MHz}, \mathrm{CDCl}_{3}\right) \delta 7.66(\mathrm{~d}, J=9.0 \mathrm{~Hz}, 1 \mathrm{H}), 7.26(\mathrm{~d}, J=8.6 \mathrm{~Hz}, 1 \mathrm{H}), 6.83(\mathrm{~d}, J=8.6 \mathrm{~Hz}, 1 \mathrm{H}), 6.25(\mathrm{~d}, J=9.4 \mathrm{~Hz} \\
1 \mathrm{H}), 5.26-5.19(\mathrm{~m}, 1 \mathrm{H}), 4.18(\mathrm{~d}, J=5.8 \mathrm{~Hz}, 2 \mathrm{H}), 3.54(\mathrm{~d}, J=7.2 \mathrm{~Hz}, 2 \mathrm{H}), 3.11(\mathrm{t}, J=6.0 \mathrm{~Hz}, 2 \mathrm{H}), 2.87-2.81(\mathrm{~m}, 9 \mathrm{H}), 1.46- \\
1.36(\mathrm{~m}, 10 \mathrm{H}) .{ }^{13} \mathrm{C} \mathrm{NMR}\left(151 \mathrm{MHz}, \mathrm{CDCl}_{3}\right) \delta 161.75,159.49,152.80,143.63,132.46,130.90,128.82,121.16,118.02,112.80 \\
108.27,68.17,56.73,54.74,52.61,38.73,30.37,28.93,26.02,25.81,23.75,22.03,18.10\end{array}$ \\
\hline
\end{tabular}




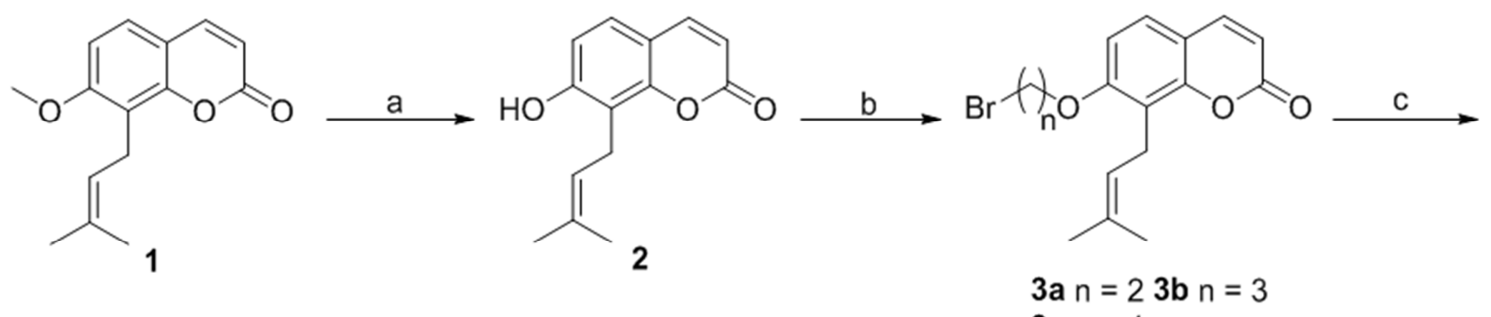<smiles>[R]Oc1ccc2ccc(=O)oc2c1CC=C(C)C</smiles>

$3 \mathrm{c} n=4$

Figure 1. Synthesis of compounds $4 a$-l. Reagents and conditions: (a) $\mathrm{BBr} r_{3}, \mathrm{DCM},-40^{\circ} \mathrm{C}$ to r.t., $12 \mathrm{~h}$; (b) $\mathrm{Br}\left(\mathrm{CH}_{2}\right)_{n} \mathrm{Br}$, anhydrous $\mathrm{K}_{2} \mathrm{CO} \mathrm{O}_{3}$, acetone, reflux, 4 h; (c) Anhydrous $\mathrm{K}_{2} \mathrm{CO}_{3}$, corresponding secondary amines, $\mathrm{CH}_{3} \mathrm{CN}$, reflux, $8 \mathrm{~h}$.

\subsection{Biological Studies}

Cytotoxic activity screening and SAR

In vitro antiproliferative activity of all the synthesized osthole derivatives was evaluated against four human cancer cell lines (MCF-7, HepG2, HCT116 and SCG7901) by using MTT method. 5-Fluorouracil (5-Fu), an effective anticancer agent, and osthole were used as positive control for comparison [16-19]. All biological results are summarized in Table 2. Form the results, it can be seen that most compounds show moderate anti-proliferation activities with $\mathrm{IC}_{50}$ values less than $200 \mu \mathrm{M}$. The structure-activity relationship (SAR) analysis indicated that, with exception of compound 41, compounds (4f-k) with substituted piperazine showed much higher anticancer activity than compounds with other secondary amines (4a-e). The piperazine substituted with a pyrimidine group seemed more favorable for the activity, as compound $4 \mathrm{k}$ showed stronger inhibition against these four cell lines than its congeners $4 \mathrm{f}-\mathrm{j}$. Lengthening the linker between osthole and the secondary amine groups could not improve the anticancer activity. For example, compound $4 \mathrm{f}$ with short linker $(n=2)$ showed more potent activity than $4 \mathrm{~g}(\mathrm{n}$ $=3)$ and $4 \mathrm{~h}(\mathrm{n}=4)$ with long linker. Compound 41 with a 4-piperidinopiperidine group showed the most potent activity in this series with $\mathrm{IC}_{50}$ values of $37.57 \mu \mathrm{M}, 25.12 \mu \mathrm{M}, 46.54$ $\mu \mathrm{M}$ and $18.39 \mu \mathrm{M}$ against SCG7901, HCT116, MCF-7 and HepG2, respectively, which was more potent than those of 5-Fu and its lead compound osthole. These results demonstrated the rationality of our molecular design.

Table 2. Cytotoxic activity $\left(I C_{50}, \mu M\right)$ of synthesized compounds 4a-l.

\begin{tabular}{|c|c|c|c|c|c|}
\hline \multirow{2}{*}{ Compd. } & \multirow{2}{*}{$\mathbf{R}$} & \multicolumn{4}{|l|}{$\mathrm{IC}_{50}(\mu \mathrm{M})^{\mathrm{a}}$} \\
\hline & & SGC7901 & HCT116 & MCF-7 & HepG2 \\
\hline $4 a$ & & $>200$ & $>200$ & $>200$ & $109.38 \pm 10.32$ \\
\hline $4 b$ & & $>200$ & $>200$ & $>200$ & $>200$ \\
\hline $4 c$ & & $>200$ & $>200$ & $>200$ & $>200$ \\
\hline $4 d$ & & $>200$ & $>200$ & $64.41 \pm 6.77$ & $115.57 \pm 10.37$ \\
\hline $4 \mathrm{e}$ & & $>200$ & $>200$ & $150.75 \pm 13.22$ & $>200$ \\
\hline $4 \mathrm{f}$ & & $90.00 \pm 8.11$ & $62.74 \pm 2.67$ & $124.46 \pm 12.19$ & $65.01 \pm 4.15$ \\
\hline $4 \mathrm{~g}$ & & $106.16 \pm 11.32$ & $120.18 \pm 9.53$ & $87.00 \pm 10.14$ & $79.92 \pm 8.16$ \\
\hline $4 \mathrm{~h}$ & & $120.12 \pm 9.75$ & $146.05 \pm 14.03$ & $120.43 \pm 12.15$ & $137.20 \pm 14.19$ \\
\hline $4 \mathrm{i}$ & & $190.78 \pm 13.32$ & $61.83 \pm 4.22$ & $>200$ & $100.61 \pm 9.12$ \\
\hline $4 j$ & & $141.72 \pm 11.12$ & $131.22 \pm 7.69$ & $80.79 \pm 6.11$ & $61.31 \pm 3.47$ \\
\hline
\end{tabular}




\begin{tabular}{|c|c|c|c|c|c|}
\hline \multirow{2}{*}{ Compd. } & \multirow{2}{*}{$\mathbf{R}$} & \multicolumn{4}{|l|}{$\mathrm{IC}_{50}(\mu \mathrm{M})^{\mathrm{a}}$} \\
\hline & & SGC7901 & HCT116 & MCF-7 & HepG2 \\
\hline $4 k$ & & $76.42 \pm 5.24$ & $65.82 \pm 5.33$ & $48.96 \pm 2.93$ & $53.79 \pm 5.15$ \\
\hline 41 & & $37.57 \pm 3.70$ & $25.12 \pm 2.19$ & $46.54 \pm 6.56$ & $18.39 \pm 2.10$ \\
\hline Osthole & - & $125.34 \pm 9.67$ & $>200$ & $98.98 \pm 7.91$ & $120.01 \pm 11.18$ \\
\hline $5-\mathrm{Fu}$ & - & $47.83 \pm 4.52$ & $65.99 \pm 9.31$ & $50.60 \pm 2.02$ & $49.84 \pm 10.03$ \\
\hline
\end{tabular}

${ }^{\text {a }}$ Data are expressed as the means $\pm \mathrm{SD}$ of three independent experiments data.

\section{Hoechst 33258 staining.}

Apoptosis plays a central role in cancer, since its induction in cancer cells is critical to a successful therapy [20-22]. It is thus believed that apoptosis assay may provide important information to preliminary investigation of the mode of action. Therefore, in the present study, compound 41 that exhibited good cytotoxic inhibition in four cancer cell lines was selected as a representative for mechanism of growth inhibition of HepG2 cells by Hoechst 33258 staining [23-25]. Hoechst 33258 is a membrane permeable blue fluorescent dye that stains the cell nucleus. Live cells with uniformly light blue nuclei were observed under fluorescence microscope after
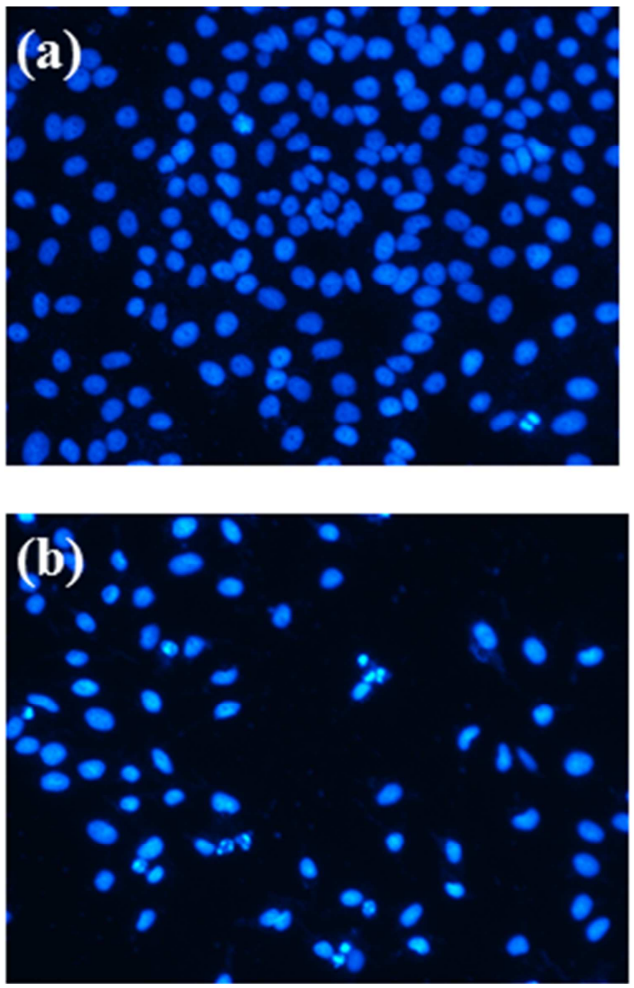

Figure 2. Hoechst 33258 staining of compound $4 \mathrm{l}$ in HepG2 cells. (a) Not treated with compound $4 \mathrm{l}$ was used as control for $48 \mathrm{~h}$ and (b, $c$ and d) treatment with compound $4 \mathrm{l}(15 \mu \mathrm{M})$ for 12,24 and $48 \mathrm{~h}$, respectively.

\section{Conclusion}

Osthole is an important natural product with antitumor bioactivity. However, the comparatively weak activity hinders its further application in clinical. In present study, with aim to improve the activity of osthole, a series of novel osthole derivatives have been designed and synthesized by connecting osthole with different secondary amine groups through treatment with Hoechst 33258, whereas apoptotic cells had bright blue nuclei because of karyopyknosis and chromatin condensation; the nuclei of dead cells could not be stained [26-28]. During the assay, HepG2 cells treated with compound 41 at $15 \mu \mathrm{M}$ from 12 to $48 \mathrm{~h}$ were stained with Hoechst 33258 . HepG2 cells not treated with the 41 were used as control for 48 h. As shown in Figure 2, HepG2 cells not treated with compound 41 were normally blue, whereas the cells treated with 41 displayed strong blue fluorescence and indicated typical apoptotic morphology after 12, 24 and $48 \mathrm{~h}$. These findings demonstrated that compound 41 could induce apoptosis against $\mathrm{HepG} 2$ cell line.
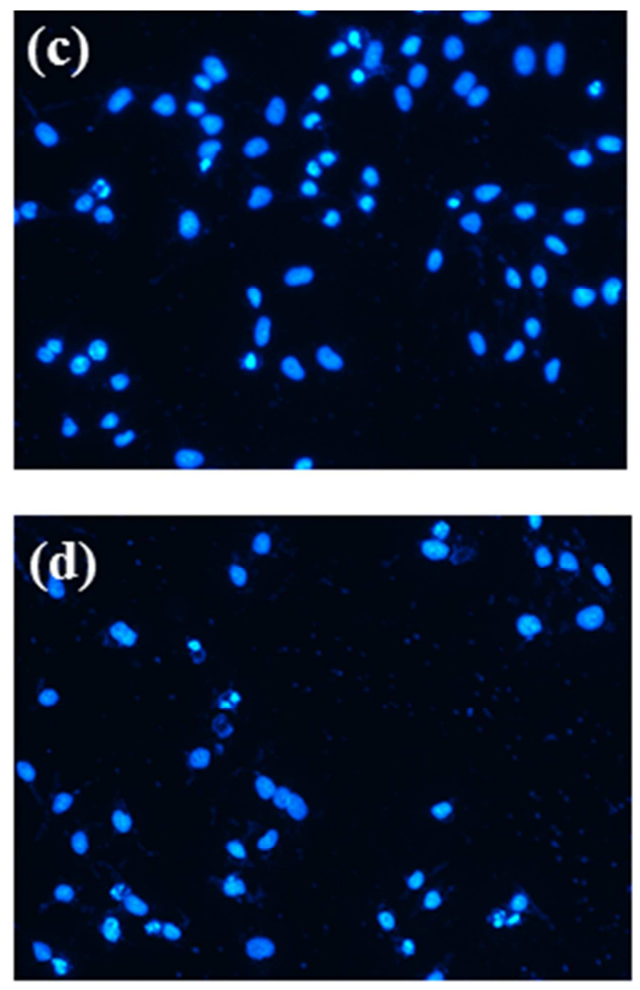

polymethene linkers. All compounds were evaluated for their cell growth inhibition activities against four cell lines (SCG7901, HCT116, MCF-7 and HepG2). The results indicated that most of compounds exhibited moderate to good anti-cancer activities with $\mathrm{IC}_{50}$ values ranging from 18.39 to $190.78 \mu \mathrm{M}$. Of these compounds, compound 41 stood out as most potent compound in this series with $\mathrm{IC}_{50}$ values of 37.57 $\mu \mathrm{M}, 25.12 \mu \mathrm{M}, 46.54 \mu \mathrm{M}$ and $18.39 \mu \mathrm{M}$ against SCG7901, 
HCT116, MCF-7 and HepG2, respectively, which was more potent than those of 5-Fu and its parent compound osthole. The SAR analysis suggested that introduction of substituted piperazine was favorable to antiproliferative activity, and compound with short linker displayed more potent activity than compound with long linker. The Hoechst 33258 staining assay revealed that compound 41 could induce cell apoptosis in HepG2 cells. As apoptosis is important for the prevention and treatment of cancer, the further study should be performed to investigate the exact pathway of compound 4l. Overall, the above results demonstrate that modification of osthole to improve its activity is feasible, and compound 41 can be considered as a novel anticancer agent for clinical development.

\section{Acknowledgements}

This research work was financially supported by the Program of the National Natural Science Foundation of China [Grant No. 81760622, 21807052]; the Program of Natural Science Foundation of Jiangxi Province of China [grant No. 20171BAB215064]; Research Fund for the Doctoral Program of Jiangxi University of Traditional Chinese Medicine [Grant No. 2015BS008]; Project from Health and Family planning Commission of Jiangxi province [Grant No. 20173013].

\section{References}

[1] Kim S. K. (2015), Handbook of Anticancer Drugs from Marine Origin, Springer international polishing, Switzerland.

[2] Salas-Vega S., Mossialos E (2017). Overestimating the Benefit of Cancer Drugs-Reply. JAMA oncol. 3: 1738-39.

[3] Wang, W., Zhang, Y., Yao, G., et al. (2018), Synthesis of new sarsasapogenin derivatives with antiproliferative and apoptotic effects in MCF-7 cells, Steroids, 131: 23-31.

[4] Ramos, P., Bentires-Alj, M (2015). Mechanism-based cancer therapy: resistance to therapy, therapy for resistance, Oncogene, 34: 3617-3626.

[5] Ozben T. (2006), Mechanisms and strategies to overcome multiple drug resistance in cancer, FEBS Lett. 580: 2903-2909.

[6] Cragg G. M., Newman D. J. (2013), Natural products: a continuing source of novel drug leads, Biochim. Biophys. Acta. 1830: 3670-3695.

[7] Kumar, M. S., Adki, K. M (2018), Marine natural products for multi-targeted cancer treatment: A future insight, Biomed. Pharmacother, 105: 233-245.

[8] Wang, Y., Zhong, J., Bai, J., et al. (2018), The Application of Natural Products in Cancer Therapy by Targeting Apoptosis Pathways, Curr. Drug Metab. 19: 739-749.

[9] Newman, D. J., Cragg, G. M., Snader, K. M. (2003), Natural products as sources of new drugs over the period 1981-2002, J. Nat. Prod. 66: 1022-1037.
[10] Yuan, R., Hou, Y., Sun, W., Yu, J., Liu, X., et al. (2017), Natural products to prevent drug resistance in cancer chemotherapy: a review, Ann N Y Acad. Sci., 1401: 19-27.

[11] Zimecki M., Artym J., Cisowski W., et. al (2009), Immunomodulatory and anti-inflammatory activity of selected osthole derivatives, Z. Naturforsch. C 64 (5-6) 361-368.

[12] Chao X., Zhou X., Zheng G., et. al (2014), Osthole induces G2/M cell cycle arrest and apoptosis in human hepatocellular carcinoma HepG2 cells. Pharmaceutical biology, 52 (5): 544-550.

[13] Zhang Z. R., Leung W. N., Cheung H. Y. (2015), Osthole: a review on its bioactivities, pharmacological properties, and potential as alternative medicine. Evidence-Based Complementary and Alternative Medicine, 2015.

[14] You L., An R., Wang X., Li Y. (2010), Discovery of novel osthole derivatives as potential anti-breast cancer treatment, Bioorg. Med. Chem. Lett. 20: 7426-7428.

[15] You L., Feng S., An R., Wang X. (2009), Osthole: a promising lead compound for drug discovery from a traditional Chinese medicine (TCM), Nat. Prod. Commun. 4: 297-302.

[16] Putnam K. P., Bombick D. W., Doolittle D. J. (2002), Evaluation of eight in vitro assays for assessing the cytotoxicity of cigarette smoke condensate, Toxicol. In Vitro 16: 599-607.

[17] Kaise, A., Ohta, K., Shirata, C., Endo, Y. (2017), Design and synthesis of $p$-carborane-containing sulfamates as multitarget anti-breast cancer agents, Bioorg. Med. Chem. 25: 6417-6426.

[18] Duan Y. C., Ma Y. C., Zhang E., et. al. (2013), Design and synthesis of novel 1,2,3-triazole-dithiocarbamate hybrids as potential anticancer agents, Eur. J. Med. Chem. 62: 11-19.

[19] Ahmed, M. F., Magdy, N. (2017), Design and Synthesis of 4-substituted Quinazolines as Potent EGFR Inhibitors with Anti-breast Cancer Activity, Anticancer Agents Med. Chem., 17: 832-838.

[20] Bunz F. (2001), Cell death and cancer therapy, Curr. Opin. Pharmacol., 1: 337-341.

[21] Guo, C., Yang, R. J., Jang, K., Zhou, X. L., Liu, Y. Z. (2017), Protective Effects of Pretreatment with Quercetin Against Lipopolysaccharide-Induced Apoptosis and the Inhibition of Osteoblast Differentiation via the MAPK and Wnt/beta-Catenin Pathways in MC3T3-E1 Cells, Cell Physiol Biochem, 43: 1547-1561.

[22] Krajarng A., Nilwarankoon S., Suksamrarn S., Watanapokasin R. (2012), Antiproliferative effect of alpha-mangostin on canine osteosarcoma cells, Res. Vet. Sci. 93: 788-794.

[23] Huang X. C., Wang M., Wang H. S., et. al (2014), Synthesis and antitumor activities of novel dipeptide derivatives derived from dehydroabietic acid, Bioorg. Med. Chem. Lett. 24: 1511-1518.

[24] Wu J. J., Ma T., Wang Z. M., Xu W. J., et. al (2017). Polycyclic xanthones via $\mathrm{pH}$-switched biotransformation of $\alpha$-mangostin catalysed by horseradish peroxidase exhibited cytotoxicity against hepatoblastoma cells in vitro. Journal of Functional Foods, 28: 205-214.

[25] Zhang, B., Huang, R., Hua, J. et al. (2016), Antitumor lignanamides from the aerial parts of Corydalis saxicola, Phytomedicine, 23: 1599-1609. 
[26] Liu M. C., Yang S. J., Jin L. H., et. al (2012), Synthesis and cytotoxicity of novel ursolic acid derivatives containing an acyl piperazine moiety, Eur. J. Med. Chem., 58: 128-135.

[27] Tian, L., Wang, X., Li, X., et al. (2016), In vitro antitumor activity of Latcripin-15 regulator of chromosome condensation 1 domain protein, Oncol. Lett., 12: 3153-3160.
[28] Rubino S., Portanova P., Albanese A. (2007), Mono- and polynuclear complexes of Pt (II) with polypyridyl ligands. Synthesis, spectroscopic and structural characterization and cytotoxic activity, J. Inorg. Biochem. 101: 1473-1482. 